\title{
Improve some of bounds for the Randić Estrada index of graphs
}

\author{
A. Jahanbani \\ Department of Mathematics \\ Azarbaijan Shahid Madani University \\ Tabriz, Iran \\ akbar.jahanbani92@gmail.com
}

\begin{abstract}
Let $G$ be a graph with $n$ vertices and let $\rho_{1}, \rho_{2}, \ldots, \rho_{n}$ be the eigenvalues of Randić matrix. The Randić Estrada index of $G$ is $R E E(G)=\sum_{i=1}^{n} e^{\rho_{i}}$. In this paper, we establish new lower and upper bounds for Randić index in terms of graph invariants such as the number of vertices and eigenvalues of graphs and improve some previously published lower bounds.
\end{abstract}

Keywords: Eigenvalue of graph, Estrada index, Randić Estrada index, Bounds.

\section{AMS subject classification: 35B40; $35 \mathrm{~K} 35$}

\section{Introduction}

Let $G=(V, E)$ be a simple undirected graph with $n$ vertices and $m$ edges. The adjacency matrix $A(G)$ of $G$ is defined by its entries as $a_{i j}=1$ if $v_{i} v_{j} \in E(G)$ and 0 otherwise. Let $\lambda_{1} \geqslant \lambda_{2} \geqslant \cdots \geqslant \lambda_{n}$ denote the eigenvalues of $A(G) . \lambda_{1}$ is called 
the spectral radius of the graph $G$. The graph invariant $E E$ defined by

$$
E E(G)=\sum_{i=1}^{n} e^{\lambda_{i}}
$$

was introduced by Estrada [7] in 2000, it is called Estrada index. Estrada index was first used to quantify the degree of folding of protein. It has also been used to measure centrality of complex networks. Applications of the Estrada index in quantum chemistry [8] and in information theory [5] can also be found in the literature. In addition to this, in [8] a connection between $E E$ and the concept of extended atomic branching was considered. An application of the Estrada index in statistical thermodynamic has also been reported [9]. Mathematical properties of the Estrada index were studied in a number of recent works ( [1], [6], [10], [11], [12], [13], [14], [20]).

For any graph $G$ of order $n$, the Randić matrix of $G, \mathbf{R}=\left(r_{i j}\right)$, is defined in [2] as follows:

$$
r_{i j}= \begin{cases}\frac{1}{\sqrt{d_{i} \times d_{j}}} & \text { if the vertices } v_{i} \text { and } v_{j} \text { of } G \text { are adjacent } \\ 0 & \text { otherwise. }\end{cases}
$$

The Randić eigenvalues $\rho_{1}, \rho_{2}, \ldots, \rho_{n}$ of the graph $G$ are the eigenvalues of its Randić matrix. Since $R(G)$ is real symmetric matrix, its eigenvalues are real number. So we can order them so that $\rho_{1} \geq \rho_{2} \geq \cdots \geq \rho_{n}$.

Then the Randić Estrada index of the graph $G$ is defined in [3] as following:

$$
\operatorname{REE}(G)=\sum_{i=1}^{n} e^{\rho_{i}}
$$

Recall that the general Randić index of a graph $G$ is defined in [4] as following:

$$
R_{\alpha}=R_{\alpha}(G)=\sum_{u v \in E(G)}\left(d_{u} d_{v}\right)^{\alpha}
$$

The general Randić index when $\alpha=-1$ is

$$
R_{-1}=R_{-1}(G)=\sum_{u v \in E(G)} \frac{1}{d_{u} d_{v}} .
$$

We make use of the following results in this paper. 
Lemma 1. [15] Let $a_{1}, a_{2}, \ldots, a_{n} \geq 0$ and $p_{1}, p_{2}, \ldots, p_{n} \geq 0$ such that $\sum_{i=1}^{n} p_{i}=1$. Then

$$
\sum_{i=1}^{n} p_{i} a_{i}-\prod_{i=1}^{n} a_{i}^{p_{i}} \geq n \Upsilon\left(\frac{1}{n} \sum_{i=1}^{n} a_{i}-\prod_{i=1}^{n} a_{i}^{\frac{1}{n}}\right) .
$$

where $\Upsilon=\min \left\{p_{1}, \ldots, p_{n}\right\}$. Equality holds if and only if $a_{1}=\cdots=a_{n}$.

Lemma 2. [18] Let $a_{1}, a_{2}, \ldots, a_{n}>0$. Then

$$
n\left(\frac{1}{n} \sum_{i=1}^{n} a_{i}-\left(\prod_{i=1}^{n} a_{i}\right)^{\frac{1}{n}}\right) \leq \Phi \leq n(n-1)\left(\frac{1}{n} \sum_{i=1}^{n} a_{i}-\left(\prod_{i=1}^{n} a_{i}\right)^{\frac{1}{n}}\right)
$$

where $\Phi=n \sum_{i=1}^{n} a_{i}-\left(\sum_{i=1}^{n} \sqrt{a_{i}}\right)^{2}$.

Lemma 3. [16] The Randić spectral radius is one.

Lemma 4. For any real $x, e^{x} \geqslant 1+x+\frac{x^{2}}{2}+\frac{x^{3}}{3}+\frac{x^{4}}{4 !}+\frac{x^{5}}{5 !}$. Equality holds if and only if $x=0$.

Let $t r$ denotes the trace of a Randić matrix and $\rho_{1}, \rho_{2}, \ldots, \rho_{n}$ be the eigenvalues of the Randić matrix $R(G)$, recall that

$$
N_{k}=\operatorname{tr}\left(R^{k}\right)=\sum_{i=1}^{n} \rho_{i}^{k}
$$

and

$$
\operatorname{REE}(G)=\sum_{i=1}^{n} \frac{N_{k}}{k !}
$$

Lemma 5. [17] Let $G$ be a graph of order $n$ and Randic matrix $R$. Then

$$
\begin{aligned}
& \operatorname{tr}(R)=0, \\
& \operatorname{tr}\left(R^{2}\right)=2 \sum_{u v \in E(G)} \frac{1}{d_{u} d_{v}}, \\
& \operatorname{tr}\left(R^{3}\right)=2 \sum_{u v \in E(G)} \frac{1}{d_{u} d_{v}} \sum_{\substack{z \in V(G) \\
z \sim u, z \sim v}} \frac{1}{d_{z}}, \\
& \operatorname{tr}\left(R^{4}\right)=\sum_{u \in V(G)}\left(\sum_{\substack{v \in V(G) \\
u \sim v}} \frac{1}{d_{u} d_{v}}\right)^{2}+\sum_{\substack{u, v \in V(G) \\
u \neq v}} \frac{1}{d_{u} d_{v}}\left(\sum_{\substack{z \in V(G) \\
z \sim u, z \sim v}} \frac{1}{d_{z}}\right)^{2} .
\end{aligned}
$$


Theorem 1. [19] Let $A_{n}(x)$ and $G_{n}(x)$ be the arithmetic and the geometric mean, respectively. Then

$$
A_{n}(x)-G_{n}(x) \geq \frac{1}{n}(\sqrt{b}-\sqrt{a})^{2}
$$

where $a=x_{1} \leq x_{2} \leq \cdots \leq x_{n}=b$.

\section{Motivation}

Given that the Estrade index is one of the first and most applications topological indices. Recently, the Randić index has come to the attention of many researchers and also, we all know that the Randić index has wide applications in chemistry and physics. Maden in [17], obtained some of bounds for Randić Estrada index and Bozkurt et al. [3], discussed on the Randić Estrada index. In this paper, we decided to improve the published bounds of the Randic Estrada index.

\section{Bounds for the Randić Estrada index}

In this section, we establish some lower bounds for the Randić Estrada index in terms of graph invariants such as the order and eigenvalues. First we present a lower bound for Randić Estrada index that this bound improve bound in Theorem 1 for $n \geq 3$.

Theorem 2. Let $G$ be a connected graph of order $n$. Then

$$
\operatorname{REE}(G) \geq e+(n-2) .
$$

Proof. Consider the following function $f(x)=(x-1)-\ln (x)$ for $x>0$. Clearly, $f$ is decreasing in $(0,1]$ and increasing in $[-1,+1)$. Hence, $f(x) \geq f(1)=0$, implying that

$$
x \geq 1+\ln x, x>0
$$

with equality if and only if $x=1$. By the definition of Randić Estrada index and 
Inequality (5), we get:

$$
\begin{aligned}
R E E(G) \geq e^{\rho_{1}}+(n-1)+\sum_{i=2}^{n} \ln e^{\rho_{i}} \\
=e^{\rho_{1}}+(n-1)+\sum_{i=2}^{n} \rho_{i} \\
=e^{\rho_{1}}+(n-1)+\sum_{i=1}^{n} \rho_{i}-\rho_{1} \\
=e^{\rho_{1}}+(n-1)-\rho_{1} .
\end{aligned}
$$

Since the function $g(x)=e^{x}+(n-1)-x$, is increasing on $D_{g}=[0,+\infty)$, using Lemma 3 we get the result.

If $G$ is bipartite, the property of R-eigenvalues of $G$ is similar to that of A-eigen values of $G$, hence $\rho_{1}=-\rho_{n}$. In the following result, we obtain a sharp lower bound of the Randić Estrada index for a bipartite graph.

Theorem 3. Let $G$ be a connected bipartite graph of order $n$. Then

$$
\operatorname{REE}(G)>e+\frac{1}{e}+(n-2) .
$$

Proof. By the definition of Randić Estrada index and Inequality (5), we have

$$
\begin{aligned}
R E E(G) & =e^{\rho_{1}}+e^{-\rho_{1}}+\sum_{i=2}^{n-1} e^{\rho_{i}} \\
& \geq 2 \cosh \rho_{1}+(n-2)+\sum_{i=2}^{n-1} \rho_{i} \\
& =2 \cosh \rho_{1}+(n-2)+\sum_{i=1}^{n} \rho_{i}+\rho_{1}-\rho_{1} \\
& =2 \cosh \rho_{1}+(n-2) .
\end{aligned}
$$

Since,

$$
f(x)=2 \cosh x+(n-2) .
$$

is an increasing function on $D_{f}=[0,+\infty)$, hence we get the result.

The bound of Theorem 3 improves the next results for all connected bipartite graphs $G$ with $\Delta(G) \geq \frac{n}{2}$. 
Theorem 4. [17] Let $G$ be a connected bipartite graph with $n$ vertices. Then

$$
R E E(G) \geq e+\frac{1}{e}+\sqrt{(n-2)^{2}+\frac{2(n-2 \Delta)}{\Delta}} .
$$

The next result presented in [17], is an immediate consequence of Theorem 2.

Corollary 1. [17] Let $G$ be a connected bipartite graph of order $n$. Then

$$
\operatorname{REE}(G) \geq e+\frac{1}{e}
$$

Now using Lemmas 4 and 5 we present a lower bound for Randić Estrada index of graphs.

Theorem 5. Let $G$ be a graph of order $n$. Then

$$
R E E(G) \geqslant \sqrt{n^{2}+n N_{2}+\frac{n N_{3}}{3}+\frac{n N_{4}}{12}+\frac{n N_{5}}{60}+\frac{N_{2} N_{3}}{6}+\frac{N_{2}^{2}}{4}} .
$$

Proof. Suppose that $\rho_{1}, \rho_{2}, \ldots, \rho_{n}$ is the spectrum of $G$. Using Lemma 4 we have

$$
\begin{aligned}
E E(G)^{2} & =\sum_{i=1}^{n} \sum_{j=1}^{n} e^{\rho_{i}+\rho_{j}} \\
& \geqslant \sum_{i=1}^{n} \sum_{j=1}^{n}\left(1+\rho_{i}+\rho_{j}+\frac{\left(\rho_{i}+\rho_{j}\right)^{2}}{2}+\frac{\left(\rho_{i}+\rho_{j}\right)^{3}}{6}+\frac{\left(\rho_{i}+\rho_{j}\right)^{4}}{24}+\frac{\left(\rho_{i}+\rho_{j}\right)^{5}}{120}\right) \\
& =\sum_{i=1}^{n} \sum_{j=1}^{n}\left(1+\rho_{i}+\rho_{j}+\frac{\rho_{i}^{2}}{2}+\frac{\rho_{j}^{2}}{2}+\rho_{i} \rho_{j}+\frac{\rho_{i}^{3}}{6}+\frac{\rho_{j}^{3}}{6}+\frac{\rho_{i}^{2} \rho_{j}}{2}+\frac{\rho_{i} \rho_{j}^{2}}{2}+\frac{\rho_{i}^{4}}{24}+\frac{\rho_{j}^{4}}{24}\right. \\
& \left.+\frac{\rho_{i}^{2} \rho_{j}^{2}}{4}+\frac{\rho_{i}^{3} \rho_{j}}{6}+\frac{\rho_{i} \rho_{j}^{3}}{6}+\frac{\rho_{i}^{5}}{120}+\frac{\rho_{j}^{5}}{120}+\frac{\rho_{i}^{4} \rho_{j}}{24}+\frac{\rho_{i} \rho_{j}^{4}}{24}+\frac{\rho_{i}^{3} \rho_{j}^{2}}{12}+\frac{\rho_{i}^{2} \rho_{j}^{3}}{12}\right) .
\end{aligned}
$$


By Equalities (1)-(4), we have the following equations:

$$
\begin{aligned}
& \sum_{i=1}^{n} \sum_{j=1}^{n}\left(\rho_{i}+\rho_{j}\right)=n \sum_{i=1}^{n} \rho_{i}+n \sum_{j=1}^{n} \rho_{j}=0 \\
& \sum_{i=1}^{n} \sum_{j=1}^{n} \rho_{i} \rho_{j}=\left(\sum_{i=1}^{n} \rho_{i}\right)^{2}=0, \\
& \sum_{i=1}^{n} \sum_{j=1}^{n}\left(\frac{\rho_{i}^{2}}{2}+\frac{\rho_{j}^{2}}{2}\right)=\frac{n}{2} \sum_{i=1}^{n} \rho_{i}^{2}+\frac{n}{2} \sum_{j=1}^{n} \rho_{j}^{2}=n N_{2}, \\
& \sum_{i=1}^{n} \sum_{j=1}^{n}\left(\frac{\rho_{i}^{3}}{6}+\frac{\rho_{j}^{3}}{6}\right)=\frac{n}{6} \sum_{i=1}^{n} \rho_{i}^{3}+\frac{n}{6} \sum_{j=1}^{n} \rho_{j}^{3}=\frac{n N_{3}}{3}, \\
& \sum_{i=1}^{n} \sum_{j=1}^{n}\left(\frac{\rho_{i}^{4}}{24}+\frac{\rho_{j}^{4}}{24}\right)=\frac{n}{24} \sum_{i=1}^{n} \rho_{i}^{4}+\frac{n}{24} \sum_{j=1}^{n} \rho_{j}^{4}=\frac{n N_{4}}{12}, \\
& \sum_{i=1}^{n} \sum_{j=1}^{n}\left(\frac{\rho_{i}^{5}}{120}+\frac{\rho_{j}^{5}}{120}\right)=\frac{n}{120} \sum_{i=1}^{n} \rho_{i}^{5}+\frac{n}{120} \sum_{j=1}^{n} \rho_{j}^{5}=\frac{n N_{5}}{60},
\end{aligned}
$$

$\sum_{i=1}^{n} \sum_{j=1}^{n}\left(\frac{\rho_{i}^{2} \rho_{j}^{3}}{12}+\frac{\rho_{i}^{3} \rho_{j}^{2}}{12}\right)=\frac{1}{12} \sum_{i=1}^{n} \sum_{j=1}^{n} \rho_{i}^{2} \rho_{j}^{3}+\frac{1}{12} \sum_{i=1}^{n} \sum_{j=1}^{n} \rho_{i}^{3} \rho_{j}^{2}=\frac{N_{2} N_{3}}{6}$

$\sum_{i=1}^{n} \sum_{j=1}^{n} \frac{\rho_{i}^{4} \rho_{j}}{24}=\frac{1}{24} \sum_{i=1}^{n} \rho_{i}^{4} \sum_{j=1}^{n} \rho_{j}=0$

$\sum_{i=1}^{n} \sum_{j=1}^{n} \frac{\rho_{i} \rho_{j}^{4}}{24}=\frac{1}{24} \sum_{i=1}^{n} \rho_{i} \sum_{j=1}^{n} \rho_{j}^{4}=0$

$\sum_{i=1}^{n} \sum_{j=1}^{n} \frac{\rho_{i}^{2} \rho_{j}^{2}}{4}=\frac{1}{4} \sum_{i=1}^{n} \rho_{i}^{2} \sum_{j=1}^{n} \rho_{j}^{2}=\frac{N_{2}^{2}}{4}$

$\sum_{i=1}^{n} \sum_{j=1}^{n} \frac{\rho_{i} \rho_{j}^{3}}{6}=\frac{1}{6} \sum_{i=1}^{n} \rho_{i} \sum_{j=1}^{n} \rho_{j}^{3}=0$

$\sum_{i=1}^{n} \sum_{j=1}^{n} \frac{\rho_{i}^{3} \rho_{j}}{6}=\frac{1}{6} \sum_{i=1}^{n} \rho_{i}^{3} \sum_{j=1}^{n} \rho_{j}=0$

$\sum_{i=1}^{n} \sum_{j=1}^{n} \frac{\rho_{i} \rho_{j}^{2}}{2}=\frac{1}{2} \sum_{i=1}^{n} \rho_{i} \sum_{j=1}^{n} \rho_{j}^{3}=0$

and

$$
\sum_{i=1}^{n} \sum_{j=1}^{n} \frac{\rho_{i}^{2} \rho_{j}}{2}=\frac{1}{2} \sum_{i=1}^{n} \rho_{i}^{2} \sum_{j=1}^{n} \rho_{j}=0 .
$$


Combining the above relations, we get

$$
R E E(G) \geqslant \sqrt{n^{2}+n N_{2}+\frac{n N_{3}}{3}+\frac{n N_{4}}{12}+\frac{n N_{5}}{60}+\frac{N_{2} N_{3}}{6}+\frac{N_{2}^{2}}{4}},
$$

as desired.

Next, we obtain a lower bound for the Randić Estrada index in terms of the order and the eigenvalues of its Randić matrix.

Theorem 6. Let $G$ be a graph with $n \geq 2$ vertices and $m$ edges. Then

$$
R E E(G) \geq 2(n-1) e^{\frac{\rho_{2}+\cdots+\rho_{n}}{2(n-1)}}+e^{\rho_{1}}-n+1 .
$$

Proof. Let $p_{1}=\frac{1}{2 n}, p_{i}=\frac{2 n-1}{2 n(n-1)}$ for $i=2, \ldots, n$ and $a_{j}=e^{\rho_{j}}$ for $j=1, \ldots, n$. Note that $\Upsilon=\min \left\{\frac{1}{2 n}, \frac{2 n-1}{2 n(n-1)}\right\}=\frac{1}{2 n}$. By Lemma 1 we have

$$
\frac{e^{\rho_{1}}}{2 n}+\frac{2 n-1}{2 n(n-1)} \sum_{i=2}^{n} e^{\rho_{i}}-\Psi \geq \frac{1}{2}\left(\frac{1}{n} \sum_{i=1}^{n} e^{\rho_{i}}-1\right)
$$

where

$$
\Psi=e^{\frac{\rho_{1}}{2 n}} \prod_{i=2}^{n} e^{\frac{\rho_{i}(2 n-1)}{2 n(n-1)}}
$$

It is easy to verify that

$$
\Psi=e^{\frac{\rho_{1}}{2 n}} \prod_{i=2}^{n} e^{\frac{\rho_{i}(2 n-1)}{2 n(n-1)}}=e^{\frac{\rho_{2}+\cdots+\rho_{n}}{2(n-1)}} .
$$

Hence,

$$
\frac{e^{\rho_{1}}}{2 n}+\frac{(2 n-1)\left(\sum_{i=1}^{n} e^{\rho_{i}}-e^{\rho_{1}}\right)}{2 n(n-1)}-\Psi \geq \frac{1}{2 n} \sum_{i=1}^{n} e^{\rho_{i}}-\frac{1}{2}
$$

Therefore,

$$
e^{\frac{\rho_{2}+\cdots+\rho_{n}}{2(n-1)}}+\frac{e^{\rho_{1}}}{2(n-1)}-\frac{1}{2} \leq \frac{1}{2(n-1)} R E E(G)
$$

Now derive a lower bound and an upper bound of the Randić Estrada index in terms of the order $n$ and the eigenvalues of its Randić matrix.

Theorem 7. Let $G$ be a graph of order $n$. Then

$$
\frac{\left(\sum_{i=1}^{n} e^{\frac{\rho_{i}}{2}}\right)^{2}-n}{n-1} \leq \operatorname{REE}(G) \leq\left(\sum_{i=1}^{n} e^{\frac{\rho_{i}}{2}}\right)^{2}-n(n-1) .
$$

Equalities holds if and only if $G$ is the empty graph $\bar{K}_{n}$. 
Proof. Setting $a_{i}=e^{\rho_{i}}$ for $i=1,2, \ldots, n$ and applying Lemma 2, we have

$$
n\left(\frac{1}{n} \sum_{i=1}^{n} e^{\rho_{i}}-\left(\prod_{i=1}^{n} e^{\rho_{i}}\right)^{\frac{1}{n}}\right) \leq n \sum_{i=1}^{n} e^{\rho_{i}}-\left(\sum_{i=1}^{n} e^{\frac{\rho_{i}}{2}}\right)^{2}
$$

Since $\sum_{i=1}^{n} \rho_{i}=0$, we get

$$
\sum_{i=1}^{n} e^{\rho_{i}}-n \leq n \sum_{i=1}^{n} e^{\rho_{i}}-\left(\sum_{i=1}^{n} e^{\frac{\rho_{i}}{2}}\right)^{2}
$$

Therefore by the definition of Randić Estrada index, we have

$$
\operatorname{REE}(G) \geq \frac{\left(\sum_{i=1}^{n} e^{\frac{\rho_{i}}{2}}\right)^{2}-n}{n-1}
$$

Again by Lemma 2, we obtain

$$
n \sum_{i=1}^{n} e^{\rho_{i}}-\left(\sum_{i=1}^{n} e^{\frac{\rho_{i}}{2}}\right)^{2} \leq n(n-1)\left(\frac{1}{n} \sum_{i=1}^{n} e^{\rho_{i}}-\left(\prod_{i=1}^{n} e^{\rho_{i}}\right)^{\frac{1}{n}}\right)
$$

By the definition of Randić Estrada index, we have

$$
R E E(G) \leq\left(\sum_{i=1}^{n} e^{\frac{\rho_{i}}{2}}\right)^{2}-n(n-1)
$$

The equality holds if and only if all $\rho_{i}$ are zero that is $G$ is $\bar{K}_{n}$.

Theorem 8. Let $G$ be a graph of order $n$. Then

$$
R E E(G) \geq\left(\sqrt{e^{\rho_{1}}}-\sqrt{e^{\rho_{n}}}\right)^{2}+n
$$

Furthermore, this bound is sharp for $\overline{K_{n}}$.

Proof. Setting $b=e^{\rho_{1}}$ and $a=e^{\rho_{n}}, x_{i}=e^{\rho_{i}}$ for $i=1,2, \ldots, n$ and applying Lemma 1, we have

$$
\begin{aligned}
\frac{R E E(G)}{n} & =\frac{1}{n} \sum_{i=1}^{n} e^{\rho_{i}} \\
& \geq \frac{1}{n}\left(\sqrt{e^{\rho_{1}}}-\sqrt{e^{\rho_{n}}}\right)^{2}+\left(\prod_{i=1}^{n} e^{\rho_{i}}\right)^{\frac{1}{n}} \\
& =\frac{1}{n}\left(\sqrt{e^{\rho_{1}}}-\sqrt{e^{\rho_{n}}}\right)^{2}+1
\end{aligned}
$$

and this leads to the desired bound. 
The bound in Theorem 8 improves the next bound when $n \geq 2$ and $\frac{1}{e^{1 / n-1}}<1$.

Theorem 9. [17] Let $G$ be a connected graph of order $n$. Then

$$
R E E(G) \geq e+\frac{n-1}{e^{\frac{1}{n-1}}}
$$

If $\Delta \geq 2$, then we have

$$
\begin{aligned}
& e+\sqrt{(n-1)+\left(1+\frac{n-2}{e^{\frac{2}{n-1}}}\right)+2\left(\frac{2 n}{\Delta}-3\right)} \\
& \leq e+\sqrt{(n-1)+(1+n-2)+2\left(\frac{2 n}{2}-3\right)} \\
& =e+\sqrt{4(n-1)} \\
& =e+2 \sqrt{n-1}
\end{aligned}
$$

and so for $n \geq 7$, we have

$$
e+2 \sqrt{n-1}<\left(\sqrt{e^{\rho_{1}}}-\sqrt{e^{\rho_{n}}}\right)^{2}+n
$$

Thus the bound in Theorem 8 improves the next bound for all graphs of order $n \geq 7$ with $\Delta(G) \geq 2$.

Theorem 10. [17] Let $G$ be a graph of order $n$ with maximum degree $\Delta$. Then

$$
R E E(G) \geq e+\sqrt{(n-1)+\left(1+\frac{n-2}{e^{\frac{2}{n-1}}}\right)+2\left(\frac{2 n}{\Delta}-3\right)} .
$$

\section{Conclusions}

In this paper, At first we obtain new bounds for Randić Estrada index and improved some of the important published bounds.

\section{References}

[1] A. Alhevaz, M. Baghipur, Y. Shang, On generalized distance Gaussian Estrada index of graphs, Symmetry. (2019), 10, 1276. 
[2] S. B. Bozkurt, A. D. Güngör, I. Gutman, A. S. Cevik, Randić Matrix and Randić Energy, MATCH Commun. Math. Comput. Chem. 64 (2010), 239-250.

[3] S. Bozkurt, D. Bozkurt, Randić energy and Randić Estrada index of a graph, Eur. J. Pure Appl. Math. 5 (2012), 88-96.

[4] B. Bollobás, P. Erdös, Graphs of extremal weights, Ars Combin. 50 (1998), $225-233$.

[5] R. Carbó-Dorca, Smooth function topological structure descriptors based on graph-spectra, J. Math. Chem. 44 (2008), 373-378.

[6] H. Deng, S. Radenković, I. Gutman, The Estrada index. In Cvetkoviacutec, D. Gutman, I. (eds.) Applications of Graph Spectra, Mathematical Institute, Belgrade (2009), 123-140.

[7] E. Estrada, Characterization of 3d molecular structure, Chem. Phys. Lett. 319 (2000), 713-718.

[8] E. Estrada, J. A. Rodríguez-Velázquez, M. Randić, Atomic branching in molecules, Int.J. Quantum Chem. 106 (2006), 823-832.

[9] E. Estrada, N. Hatano, Statistical-mechanical approach to subgraph centrality in complex networks. Chem. Phys. Lett. 439 (2007), 247-251.

[10] J.A. de la Peã, I. Gutman, J. Rada, Estimating the Estrada index. Linear Algebra Appl. 427 (2007), 70-76.

[11] I. Gutman, Lower bounds for Estrada index. Publ. Inst. Math. (Beograd) . 83 (2008), 1-7.

[12] N. Jafari. Rad, A. Jahanbani, D. A. Mojdeh, Tetracyclic Graphs with Maximal Estrada Index, Discrete Mathematics, Algorithms and Applications. 9 (2017), 1750041. doi.org/10.1142/S1793830917500410

[13] N. Jafari. Rad, A. Jahanbani, I. Gutman, Zagreb energy and Zagreb estrada index of graphs, Match. Commun. Math. Comput. Chem. 79 (2018), 371-386.

[14] N. Jafari. Rad, A. Jahanbani, R. Hasni, Pentacyclic Graphs with Maximal Estrada Index, Ars Combin. 133 (2017), 133-145. 
[15] J. Li, J. Guo, W. Shiu, S. Bozkurt Altinda, D. Bozkurt, Bounding the sum of powers of normalized Laplacian eigenvalues of a graph, Applied Mathematics and Computation. 324 (2018), 82-92.

[16] B. Liu, Y. Huang, J. Feng, A note on the Randić spectral radius, MATCH Commun. Math. Comput. Chem. 68 (2012), 913-916.

[17] A. D. Maden, New bounds on the incidence Energy, Randić energy and Randić Estrada index, MATCH Commun. Math. Comput. Chem. 74 (2015), 367-387.

[18] P. B. Sarasija, R. Binthiya, Bounds on the Seidel Energy of Strongly Quotient Graphs, Journal of Chemical and Pharmaceutical Sciences. 10 (2007), 1-4.

[19] S. Simić, On a class of self-improving inequalities, Kragujevac J. Math. 37 (2013), 269-273.

[20] Y. Shang, Lower bounds for Gaussian Estrada index of graphs. Symmetry. (2018), 10, 325. 